\title{
Weakly-supervised Text Classification Based on Keyword Graph
}

\author{
Lu Zhang $^{1 *}$ Jiandong Ding $^{2} \quad$ Yi Xu $^{1 *}$ Yingyao Liu $^{2} \quad$ Shuigeng Zhou ${ }^{1 \dagger}$ \\ ${ }^{1}$ Shanghai Key Lab of Intelligent Information Processing, and School of \\ Computer Science, Fudan University, China \\ ${ }^{2}$ Alibaba Group \\ \{1_zhang19, jdding, yxu17, sgzhou\}@fudan.edu.cn
}

\begin{abstract}
Weakly-supervised text classification has received much attention in recent years for it can alleviate the heavy burden of annotating massive data. Among them, keyword-driven methods are the mainstream where user-provided keywords are exploited to generate pseudolabels for unlabeled texts. However, existing methods treat keywords independently, thus ignore the correlation among them, which should be useful if properly exploited. In this paper, we propose a novel framework called Class $K G$ to explore keyword-keyword correlation on keyword graph by GNN. Our framework is an iterative process. In each iteration, we first construct a keyword graph, so the task of assigning pseudo labels is transformed to annotating keyword subgraphs. To improve the annotation quality, we introduce a self-supervised task to pretrain a subgraph annotator, and then finetune it. With the pseudo labels generated by the subgraph annotator, we then train a text classifier to classify the unlabeled texts. Finally, we re-extract keywords from the classified texts. Extensive experiments on both long-text and short-text datasets show that our method substantially outperforms the existing ones.
\end{abstract}

\section{Introduction}

Text classification is one of the most fundamental tasks in natural language processing (NLP). In real-world scenarios, labeling massive texts is timeconsuming and expensive, especially in some specific areas that need domain experts to participate. Weakly-supervised text classification (WTC) has received much attention in recent years because it can substantially reduce the workload of annotating massive data. Among the existing methods, the mainstream form is keyword-driven (Agichtein and Gravano, 2000; Riloff et al., 2003; Kuipers

\footnotetext{
*The author did most work during internship at Alibaba.

${ }^{\dagger}$ Correspondence author
}

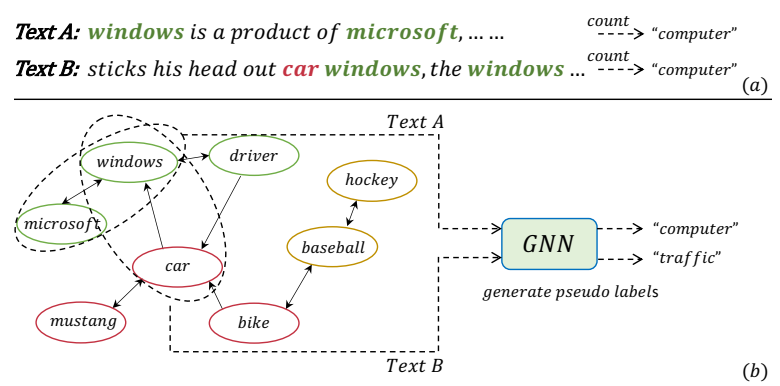

Figure 1: (a) Existing methods do not consider the correlation among keywords, which will generate wrong pseudo-label for text B. (b) Our method exploits the correlation among keywords by GNN over a keywordgraph, and converts the task of assigning pseudo-labels for unlabeled texts to annotating subgraphs, which leads to much better performance.

et al., 2006; Meng et al., 2018, 2019, 2020; Mekala and Shang, 2020; Wang et al., 2021; Shen et al., 2021), where the users need only to provide some keywords for each class. Such class-relevant keywords are then used to generate pseudo-labels for unlabeled texts.

Keyword-driven methods usually follow an iterative process: generating pseudo-labels using keywords, building a text classifier, and updating the keywords or self-training the classifier. Among them, the most critical step is generating pseudo-labels. Most existing methods generate pseudo-labels by counting keywords, with which the pseudo-label of a text is determined by the category having the most keywords in the text.

However, one major drawback of these existing methods is that they treat keywords independently, thus ignore their correlation. Actually, such correlation is important for the WTC task if properly exploited, as a keyword may implies different categories when it co-occurs in texts with other different keywords. As shown in Fig. 1, suppose the users provide keywords "windows" and "microsoft" for class "computer" and "car" for class "traffic". 
When "windows" and "microsoft" appear in text A, the "windows" means operating system, and text A should be given a pseudo-label of "computer". However, when "windows" meets "car" in text B, the "windows" means the windows of a car and text B should be given a pseudo-label "traffic". With previous simple keyword counting, text A can get a correct pseudo-label, but text B cannot. Therefore, treating keywords independently is problematic.

In this paper, we solve the above problem with a novel iterative framework called ClassKG (the abbreviation of Classification with Keyword $\boldsymbol{G r a p h}$ ) where the keyword-keyword relationships are exploited by GNN on keyword graph. In our framework, the task of assigning pseudo-labels to texts using keywords is transformed into annotating keyword subgraphs. Specifically, we first construct a keyword graph $\mathcal{G}$ with all provided keywords as nodes and each keyword node updates itself via its neighbors. With $\mathcal{G}$, any unlabeled text $T$ corresponds to a subgraph $\mathcal{G}_{\mathcal{T}}$ of $\mathcal{G}$, and assigning a pseudo-label to $T$ is converted to annotating subgraph $\mathcal{G}_{\mathcal{T}}$. To accurately annotate subgraphs, we adopt a paradigm of first self-supervised training and then finetuning. The keyword information is propagated and incorporated contextually during keyword interaction. We design a self-supervised pretext task that is relevant to the downstream task, with which the finetuning procedure is able to generate more accurate pseudo-labels for unlabeled texts. Texts that contains no keywords are ignored. With the pseudo-labels, we train a text classifier to classify all the unlabeled texts. And based on the classification results, we re-extract the keywords, which are used in the next iteration.

Furthermore, we notice that some existing methods employ simple TF-IDF alike schemes for reextracting keywords, which makes the extracted keywords have low coverage and discrimination over the unlabeled texts. Therefore, we develop an improved keyword extraction algorithm that can extract more discriminative keywords to cover more unlabeled texts, with which more accurate pseudolabels can be inferred.

In summary, our contributions are as follows:

- We propose a new framework ClassKG for weakly supervised text classification where the correlation among different keywords is exploited via GNN over a keyword graph, and the task of assigning pseudo-labels for unlabeled texts is transformed into annotating key- word subgraphs on the keyword graph.

- We design a self-supervised training task on the keyword graph, which is relevant to the downstream task and thus can effectively improve the accuracy of subgraph annotating.

- We conduct extensive experiments on both long text and short text benchmarks. Results show that our method substantially outperforms the existing ones.

\section{Related Work}

Here we review the related works, including weakly-supervised text classification and selfsupervised learning.

\subsection{Weakly-Supervised Text Classification}

Weakly-supervised text classification (WTC) aims to use various weakly supervised signals to do text classification. Weak supervision signals used by existing methods includes external knowledge base (Gabrilovich et al., 2007; Chang et al., 2008; Song and Roth, 2014; Yin et al., 2019), keywords (Agichtein and Gravano, 2000; Riloff et al., 2003; Kuipers et al., 2006; Tao et al., 2015; Meng et al., 2018, 2019, 2020; Mekala and Shang, 2020; Wang et al., 2021; Shen et al., 2021) and heuristics rules (Ratner et al., 2016, 2017; Badene et al., 2019; Shu et al., 2020). In this paper, we focus on keyword-driven methods. Among them, WeSTClass (Meng et al., 2018) introduces a self-training module that bootstraps on real unlabeled data for model refining. WeSHClass (Meng et al., 2019) extends WeSTClass to hierarchical labels. LOTClass (Meng et al., 2020) uses only label names as the keywords. Conwea (Mekala and Shang, 2020) leverages contextualized corpus to disambiguate keywords. However, all these methods treat keywords independently, so ignore their correlation, which is actually useful information for generating pseudo-labels. Different from the existing methods, we exploit keyword correlation by applying GNN to a keyword graph, which can significantly boost the quality of pseudo-labels.

\subsection{Self-supervised Learning}

Self-supervised learning exploits internal structures of data and formulates predictive tasks to learn good data representations. The key idea is to define a pretext task and generate surrogate training samples automatically to train a model. A wide range 
of pretext tasks have been proposed. For images, self-supervised strategies include predicting missing parts of an image (Pathak et al., 2016), patch orderings (Noroozi and Favaro, 2016) and instance discrimination (He et al., 2020). For texts, the tasks can be masked language modeling (Devlin et al., 2019), sentence order prediction (Lan et al., 2020) and sentence permutation (Lewis et al., 2020). For graphs, the pretext tasks can be contextual property prediction (Rong et al., 2020), attribute and edge generation (Hu et al., 2020b).

However, up to now there are only a few works of self-supervised learning for subgraph representation (Jiao et al., 2020; Qiu et al., 2020), where contrastive loss is used for subgraph instance discrimination, and the downstream tasks they serve are mainly whole graph classification or node classification, instead of subgraph classification. Here, we design a new pretext task based on the keyword graph to learn better representations of keyword subgraphs, with which the accuracy of pseudo-label generation is improved, and consequently classification performance is boosted.

\section{Method}

\subsection{Problem Definition}

The input data contains two parts: a) A set of userprovided initial keywords $S=\left\{S_{1}, S_{2}, \ldots S_{C}\right\}$ for $C$ categories, where $S_{i}=\left\{w_{1}^{i}, w_{2}^{i}, \ldots, w_{k_{i}}^{i}\right\}$ denotes the $k_{i}$ keywords of category $i, \mathrm{~b}$ ) A set of $n$ unlabeled texts $U=\left\{U_{1}, U_{2}, \ldots, U_{n}\right\}$ falling in $C$ classes. Our aim is to build a text classifier and assign labels to the unlabeled texts $U$.

\subsection{Framework}

Fig. 2 is the framework of our method, which follows an iterative paradigm. In each iteration, we first build a keyword graph $\mathcal{G}$ based on the cooccurrence relationships between keywords from all classes. Each keyword node aggregates information from its neighbors. Here, an unlabeled text corresponds to a subgraph of $\mathcal{G}$ and annotating unlabeled texts is converted to annotating the corresponding subgraphs. To train a high-quality subgraph annotator $\mathcal{A}$, we first train $\mathcal{A}$ with a designed self-supervised task on $\mathcal{G}$, then finetune it with noisy labels. After that, unlabeled texts containing keywords are mapped to subgraphs, which are annotated by $\mathcal{A}$ to generate pseudo-labels. With the pseudo-labels, we train a text classifier to classify all unlabeled texts. Based on the classification results, keywords are re-extracted and updated for the next iteration, until the keywords change little.

\subsection{Keyword Graph Construction}

To model the relationships among keywords, we construct a keyword graph $\mathcal{G}$ by representing keywords as vertices and co-occurrences between keywords as edges, denoted as $\mathcal{G}=(\mathcal{V}, \mathcal{E})$.

For vertices $\mathcal{V}$, the embedding of a node is initialized with vector $x_{v}=\left[v_{\text {class }} ; v_{\text {index }}\right] \in \mathbb{R}^{C+|\mathcal{V}|}$, where $v_{\text {class }}$ is the one-hot embedding of keyword class, $v_{\text {index }}$ is the one-hot embedding of keyword index, and $C$ is the number of classes.

For edges $\mathcal{E}$, if keywords $w_{i}$ and $w_{j}$ occur in an unlabeled text in order, there exists a directed edge from $w_{i}$ to $w_{j}$. Meanwhile, we take their cooccurrences $F_{i j}$ in unlabeled texts as edge attribute. Considering the limited number of keywords contained in a text, we do not use any sliding window to limit the number of edges.

With the keyword graph $\mathcal{G}$, keyword feature information is propagated and aggregated by GNN.

\subsection{Subgraph Annotator Training}

With the keyword graph, an unlabeled text is converted into a subgraph of $\mathcal{G}$. Specifically, the keywords in a text hit a set of vertices in $\mathcal{G}$. The subgraph is the induced subgraph of the hit vertices in $\mathcal{G}$. Assigning pseudo labels to unlabeled texts is equivalent to annotating the corresponding subgraphs, which is a graph-level classification problem. In practice, we employ graph isomorphism network (GIN) (Xu et al., 2019) as our subgraph annotator to perform node feature propagation and subgraph readout. The keyword feature is propagated and aggregated as follows:

$h_{v}^{(k)}=M L P^{(k)}\left(\left(1+\varepsilon^{(k)}\right) \cdot h_{v}^{(k-1)}+\sum_{u \in N(v)} h_{u}^{(k-1)}\right)$

where $h_{v}^{(k)}$ denotes the representation of node $v$ after the $k^{t h}$ update. $M L P^{(k)}$ is a multi-layer perceptron in the $k^{\text {th }}$ layer. $\varepsilon$ is a learnable parameter. $N(v)$ denotes all the neighbors of node $v$. Then, we perform readout to obtain subgraph representation:

$$
h_{G}=\operatorname{CONCAT}\left(\operatorname{SUM}\left(\left\{h_{v}^{(k)} \mid v \in G\right\}\right) \mid k=0 \ldots K\right)
$$

GIN concatenates the sum of all node features from the same layer as the subgraph representation.

To train a subgraph annotator with high annotating accuracy, we first train a GIN via a designed self-supervised task, then finetune it. 


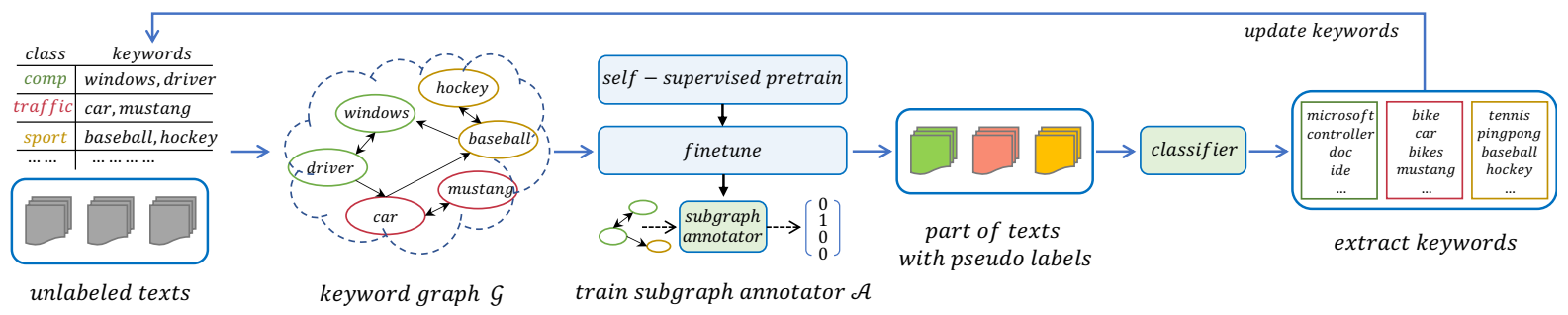

Figure 2: Our framework follows an iterative paradigm. In each iteration, we first build a keyword graph $\mathcal{G}$, with which unlabeled texts corresponds to subgraphs of $\mathcal{G}$, and assigning pseudo-labels to texts is transformed to annotating the corresponding subgraphs. To train the subgraph annotator, we design a self-supervised pretext task, and finetune it. Then, a text classifier is trained with the pseudo-labels. Based on the classification results of the classifier, keywords are re-extracted and updated for the next iteration.

\subsubsection{Self-supervised Training on Graph}

As mentioned in previous self-supervised learning works (Hu et al., 2020a), a successful pre-training needs examples and target labels that are correlated with the downstream task of interest. Otherwise, it may harm generalization, which is known as negative transfer. Considering that the downstream task is a graph-level classification, we design a graph-level self-supervised task, which is highly relevant to subgraph annotation. Our selfsupervised method is shown in Alg. 1, where the subgraph annotator $\mathcal{A}$ learns to predict the class of the start point of a random walk and the subgraph derived from the random walk is similar to the subgraph generated by an unlabeled text.

To begin with, we randomly sample a keyword $w_{r}$ from class $C_{r}$ as the start point of a random walk. The number of random walk steps follows the same Gaussian distribution $\mathcal{N}\left(u_{s}, \sigma_{s}^{2}\right)$ as that of the number of keywords appearing in an unlabeled text in $U$. Therefore, we estimate the parameters of the Gaussian distribution $u_{s}, \sigma_{s}^{2}$ based on $U$ as follows:

$$
\begin{aligned}
u_{s} & =\frac{1}{n} \sum_{i}^{n} k f\left(U_{i}\right) \\
\sigma_{s}^{2} & =\frac{1}{n-1} \sum_{i}^{n}\left[k f\left(U_{i}\right)-u_{s}\right]^{2}
\end{aligned}
$$

where $k f\left(U_{i}\right)$ is the number of keywords contained in text $U_{i}$. Then, we can sample the length $L$ of random walk from distribution $\mathcal{N}\left(u_{s}, \sigma_{s}^{2}\right)$. The probability of walking from node $w_{i}$ to node $w_{j}$ is derived from the co-occurrence frequency by

$$
p_{i j}=\frac{F_{i j}}{\sum_{w_{k} \in N\left(w_{i}\right)} F_{i k}}
$$

where $F_{i k}$ is the co-occurrence frequency of $w_{i}$ followed by $w_{k} . N\left(w_{i}\right)$ is the neighbors set of $w_{i}$.

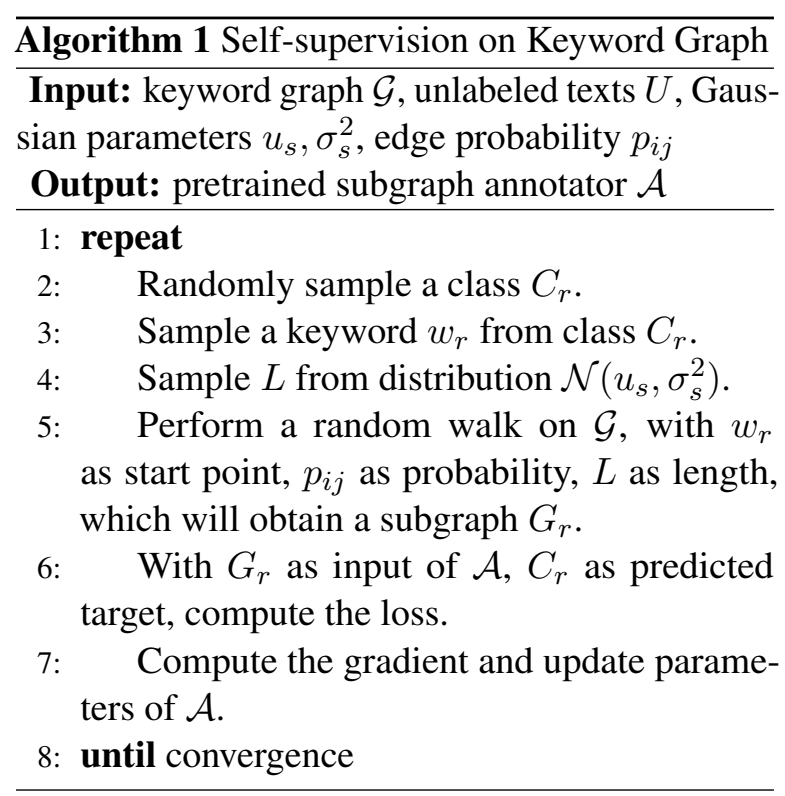

Then, we start from node $w_{r}$ to perform a $L$ step random walk. In each step, $p_{i j}$ determines the probability of jumping from $w_{i}$ to neighbor $w_{j}$. At the end of random walk, we obtain a subgraph $G_{r}$, which is the induced subgraph of the traversed nodes in the keyword graph $\mathcal{G}$.

Our self-supervised task is designed to take $G_{r}$ as the input of $\mathcal{A}$ and make $\mathcal{A}$ learn to predict the class of start point $w_{r}$. The loss function is defined as the negative log likelihood of $C_{r}$ :

$$
\mathcal{L}_{S S L}=-\sum_{r \in \text { rand }} C_{r} \log \left(\mathcal{A}\left(G_{r}\right)\right)
$$

\subsubsection{Finetuning}

After pre-training the subgraph annotator $\mathcal{A}$, we finetune it for a few epochs. The labels of finetuning are generated by voting as follows:

$$
\hat{y}_{i}=\arg \max _{k}\left\{\sum_{j} t f\left(w_{j}, U_{i}\right) \mid \forall\left(w_{j} \in S_{k}\right)\right\}
$$


where $t f\left(w_{j}, U_{i}\right)$ denotes the term-frequency (TF) of keyword $w_{j}$ in text $U_{i}$. The loss function is defined as follows:

$$
\mathcal{L}_{F T}=-\sum_{i=1}^{n}\left(k f\left(U_{i}\right)>0\right) \hat{y}_{i} \log \left(\mathcal{A}\left(G_{i}\right)\right)
$$

where $G_{i}$ is the subgraph of text $U_{i}$. Note that the number of epochs for fine-tuning cannot be too large, otherwise it may degenerate into voting.

\subsection{Text Classifier}

After training the subgraph annotator $\mathcal{A}$, we use it to annotate all the unlabeled texts $U$ and generate pseudo-labels, which are used to train a text classifier. Texts containing no keywords are ignored. Our framework is compatible with any text classifier. We use Longformer (Beltagy et al., 2020) as the long text (document) classifier and BERT (Devlin et al., 2019) as the short text (sentence) classifier. Following previous works (Meng et al., 2018, 2020), we self-train (Rosenberg et al., 2005) the classifier on all unlabeled texts. The predicted labels for all unlabeled texts by the text classifier are then used to re-extract keywords.

\subsection{Keywords Extraction}

Considering that the coverage and accuracy of userprovided keywords are limited, we re-extract keywords based on the predictions of the text classifier in each iteration. Existing methods use indicators such as term frequency (TF), inverse document frequency (IDF) and their combinations (Mekala and Shang, 2020) to rank words, and a few top ones are taken as keywords. However, they treat all indicators equally, and are prone to select common and low-information words. Here, we employ an improved TF-IDF scheme, which increases the significance of IDF to reduce the scores of common words. The score of word $w_{i}$ in class $C_{k}$ is evaluated as follows:

$$
Q\left(w_{i}, C_{k}\right)=T F\left(w_{i}, C_{k}\right) \cdot \operatorname{IDF}\left(w_{i}\right)^{M}
$$

Above, $M$ is a hyperparameter. According to the score, we select the top $Z$ words in each category as the keywords for the next iteration.

To determine whether the model has converged, we define the change of keywords as follows:

$$
\Delta=\frac{\left|S^{T_{i}}-S^{T_{i}} \cap S^{T_{i-1}}\right|}{\left|S^{T_{i}}\right|}
$$

where $S^{T_{i}}$ is the keywords set of the $i^{t h}$ iteration. If $\Delta<\epsilon$ (a hyperparameter), the iteration stops.

\begin{tabular}{c|c|c|c}
\hline Datasets & \# Texts & \# Classes & Avg Len \\
\hline 20News & 18,846 & 20(F) or 7 (C) & 400 \\
NYT & 13,081 & 26(F) or 5 (C) & 778 \\
AG News & 127,600 & 4 & 54 \\
DBPedia & 630,000 & 14 & 70 \\
IMDB & 50,000 & 2 & 297 \\
Amazon & $4,000,000$ & 2 & 104 \\
\hline
\end{tabular}

Table 1: Dataset statistics. 'F': Fine. 'C': Coarse

\section{Experiments}

\subsection{Datasets}

Different from existing works that do experiments only on long texts or short texts, we verify our method on both long and short texts. For long texts, we use two news datasets: The 20 Newsgroups and The New York Times, both of which are news articles and can be partitioned into fine-grained classes and coarse-grained classes. For short texts, we use four benchmark datasets: AG News (Zhang et al., 2015), DBPedia (Lehmann et al., 2014), IMDB (Maas et al., 2011) and Amazon (McAuley and Leskovec, 2013). Tab. 1 gives the statistics of the datasets. The initial keywords and evaluation settings follow previous works. For long texts, the initial keywords follow Conwea (Mekala and Shang, 2020) and evaluation results on the entire datasets are reported. For short texts, we follow LOTClass (Meng et al., 2020) and use the label names as initial keywords. The evaluation is performed on the test set. For all classes, we use no more than four keywords per category.

\subsection{Compared Methods}

We compare our method with a wide range of weakly-supervised text classification methods: 1) IR-TF-IDF evaluates the relevance between documents and labels by aggregated TF-IDF values of keywords. Documents are assigned labels based on their relevance to labels. 2) Dataless (Chang et al., 2008) maps label names and documents to the same space of Wikipedia concepts. Documents are classified by the semantic similarity with each label. 3) Word2Vec (Mikolov et al., 2013) first learns the word representations in the corpus and label representations are generated by aggregating the vectors of keywords. Each document is labeled with the most similar label. 4) Doc2Cube (Tao et al., 2015) leverages label names as supervision signals and performs joint embedding of labels, terms and documents to uncover their semantic sim- 


\begin{tabular}{|c|c|c|c|c|c|c|c|c|}
\hline \multirow{3}{*}{ Methods } & \multicolumn{4}{|c|}{20 Newsgroup } & \multicolumn{4}{|c|}{ NYT } \\
\hline & \multicolumn{2}{|c|}{ Fine-grained } & \multicolumn{2}{|c|}{ Coarse-grained } & \multicolumn{2}{|c|}{ Fine-grained } & \multicolumn{2}{|c|}{ Coarse-grained } \\
\hline & Micro-F1 & Macro-F1 & Micro-F1 & Macro-F1 & Micro-F1 & Macro-F1 & Micro-F1 & Macro-F1 \\
\hline IR-TF-IDF & 0.53 & 0.52 & 0.49 & 0.48 & 0.56 & 0.54 & 0.65 & 0.58 \\
\hline Dataless & 0.61 & 0.53 & 0.50 & 0.47 & 0.59 & 0.37 & 0.71 & 0.48 \\
\hline Word2Vec & 0.33 & 0.33 & 0.51 & 0.45 & 0.69 & 0.47 & 0.92 & 0.83 \\
\hline Doc2Cube & 0.23 & 0.23 & 0.40 & 0.35 & 0.67 & 0.34 & 0.71 & 0.38 \\
\hline WeSTClass & 0.49 & 0.46 & 0.53 & 0.43 & 0.50 & 0.36 & 0.91 & 0.84 \\
\hline ConWea & 0.65 & 0.64 & 0.62 & 0.57 & 0.91 & 0.79 & 0.95 & 0.89 \\
\hline ClassKG (Ours) & 0.78 & 0.77 & 0.80 & 0.75 & 0.92 & 0.80 & 0.96 & 0.83 \\
\hline
\end{tabular}

Table 2: Performance comparison on long text datasets with fine-grained and coarse-grained labels.

ilarities. 5) BERT count simply counts keywords to generate pseudo labels for training BERT. 6) WeSTClass (Meng et al., 2018) generates pseudo documents to train a classifier and bootstraps the model with self-training. 7) LOTClass (Meng et al., 2020) utilizes only label names to perform classification. They use pre-trained LM to find class-indicative words and generalizes the model via self-training. 8) ConWea (Mekala and Shang, 2020) leverages BERT to generate contextualized representations of words, which is further utilized to train the classifier and expand seed words.

\subsection{Experimental Settings}

The training and evaluation are performed on NVIDIA RTX 2080Ti. In the subgraph annotator, we use a three-layer GIN (Xu et al., 2019). We first train it with our self-supervised task $10^{6}$ iterations and then finetune it 10 epochs. We set the batch size of self-supervision/finetuning to 50/256. In classifier training, we set the batch size to $4 / 8$ for long/short texts. Both the subgraph annotator and the text classifier use AdamW (Loshchilov and Hutter, 2019) as optimizer. Their learning rates are 1e-4 and 2e-6, respectively. The classifier uses bert-base-uncased for short texts and longformerbase-4096 for long texts. For keywords extraction, we select top 100 keywords per class in each iteration. The hyperparameter $M$ is set to 4 . The keywords set change threshold $\epsilon$ is set to 0.1 . Our code has already been released. ${ }^{1}$

\subsection{Performance Comparison}

Long text datasets. The evaluation results are summarized in Table 2. Since the datasets are imbalanced, we use micro-f1 and macro-f1 as evalua-

\footnotetext{
${ }^{1}$ https://github.com/zhanglu-cst/ ClassKG
}

\begin{tabular}{c|c|c|c|c}
\hline Methods & AG News & DBPedia & IMDB & Amazon \\
\hline Dataless & 0.696 & 0.634 & 0.505 & 0.501 \\
BERT count & 0.752 & 0.722 & 0.677 & 0.654 \\
WeSTClass & 0.823 & 0.811 & 0.774 & 0.753 \\
LOTClass & 0.864 & 0.911 & 0.865 & 0.916 \\
ClassKG (Ours) & $\mathbf{0 . 8 8 8}$ & $\mathbf{0 . 9 8 0}$ & $\mathbf{0 . 8 7 4}$ & $\mathbf{0 . 9 2 6}$ \\
\hline
\end{tabular}

Table 3: Comparison on short text datasets.

tion metrics. As we can see, our method achieves SOTA, and outperforms existing weakly supervised methods in most cases. On 20Newsgroup, which is a much harder dataset, our method exceeds SOTA for all metrics by a large margin. The gap is $13 \%$ in fine-grained classification and $18 \%$ in coarsegrained classification. Although the NYT dataset is relatively simple, our model still has advantage on three of the four metrics: achieves over $1 \%$ improvement on 3 metrics, degrades a little only on macro-f1 of coarse-grained, due to the extreme imbalance of categories.

Short text datasets. Results of short text datasets are shown in Table 3. We follow previous works (Meng et al., 2020) to use accuracy as the metric. We can see that our method outperforms SOTA on all datasets, especially for DBPedia, the improvement is up to $6.9 \%$. With only label names as initial keywords, our method achieve almost $90 \%$ accuracy on all datasets.

\subsection{Ablation Study}

Here, we check the effects of various components and parameters in our framework. Experiments are conducted on $20 \mathrm{News}$ with fine-grained labels.

\subsubsection{Effectiveness of Subgraph Annotator}

To verify the effectiveness of subgraph annotator $\mathcal{A}$, we compare the results w/wo subgraph annotator $\mathcal{A}$ 


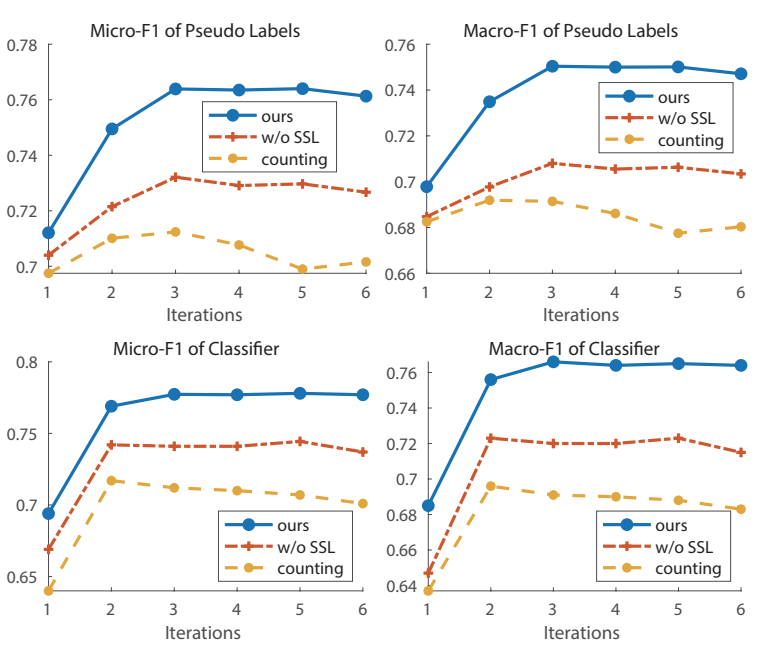

Figure 3: Comparison with the case without selfsupervised learning (SSL) and the case using counting. Results of quality of pseudo labels (top) and classification (down) in the first 6 iterations are shown.

and w/wo self-supervised learning (SSL). For the case without $\mathcal{A}$, we use keyword counting to generate pseudo-labels, which is widely used in previous works. For the case without self-supervision, we directly finetune the subgraph annotator without self-supervised training. The results of the first 6 iterations are illustrated in Fig. 3.

We can see that 1) our method with all components performs much better than the other cases, proving the effectiveness of exploiting the correlation among keywords. 2) For the case using keyword counting, since the correlation among keywords is ignored, the micro/macro-f1 of pseudo labels is the worst, which leads to the worst classification performance. 3) For the case with finetuning but no self-supervised learning, it outperforms keyword counting by $2.5 \%$ and $1.7 \%$ on micro-f1 and macro-f1 of pseudo labels in the $6^{\text {th }}$ iteration, respectively, which further leads to $3.6 \%$ and $3.2 \%$ gain on micro/macro-f1 of classification performance. 4) Our self-supervised learning task can boost performance, exceeding the case without SSL by a large margin of $3.5 \%$ and $4.4 \%$ in terms of micro/macro-f1 of pseudo labels, and $4.0 \%$ and $4.9 \%$ of classification performance.

\subsubsection{Subgraph Annotator Implementation}

We use different GNNs to implement the subgraph annotator, including GCN (Kipf and Welling, 2017), GAT (Veličković et al., 2018) and GIN (Xu et al., 2019). For GCN and GAT, we readout the subgraph by averaging all node features in the last layer. For fair comparison, all GNNs set the layer
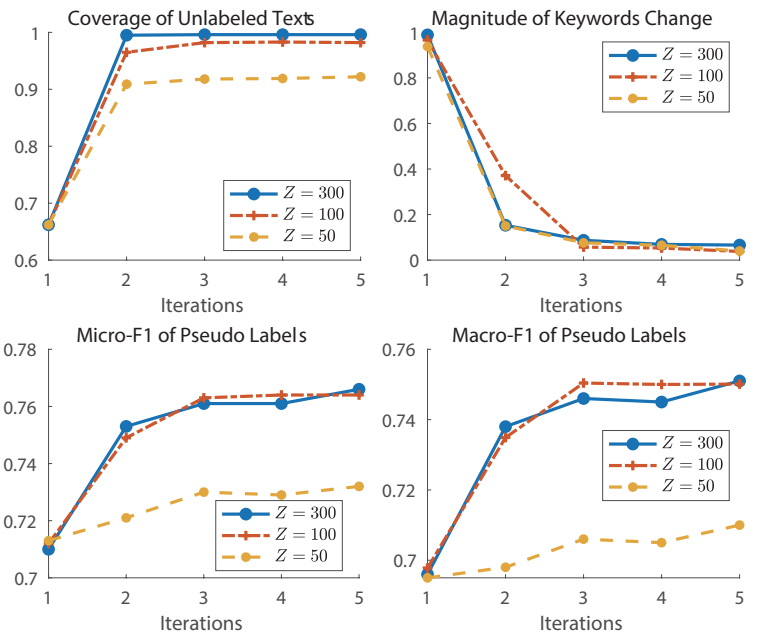

Macro-F1 of Pseudo Labels

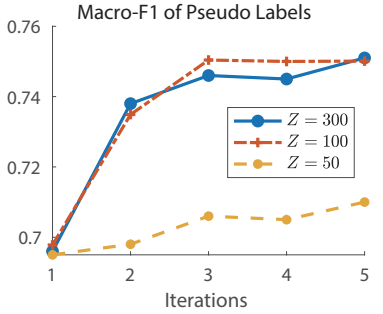

Figure 4: Effect of keywords number per class $Z$ on the coverage of unlabeled texts, the change of keywords, and the quality of pseudo labels.

number to 3. Performance comparison is given in Table 4. We can see that the performance of subgraph annotator is highly related to the selected GNN model, and a more powerful GNN model will lead to higher annotation accuracy.

\begin{tabular}{c|cccccc}
\hline Metrics & GNN / Iter & 1 & 2 & 3 & 4 & 5 \\
\hline \multirow{3}{*}{ Micro-F1 } & GCN & 0.700 & 0.712 & 0.742 & 0.743 & 0.743 \\
& GAT & 0.703 & 0.735 & 0.753 & 0.753 & 0.753 \\
& GIN & 0.712 & 0.750 & 0.764 & 0.764 & 0.764 \\
\hline \multirow{3}{*}{ Macro-F1 } & GCN & 0.684 & 0.697 & 0.726 & 0.726 & 0.726 \\
& GAT & 0.689 & 0.719 & 0.737 & 0.737 & 0.737 \\
& GIN & 0.698 & 0.735 & 0.750 & 0.750 & 0.750 \\
\hline
\end{tabular}

Table 4: Performance comparison among different subgraph annotator implementations.

\subsubsection{Effect of the Number of Keywords}

Here, we check the effect of the number of extracted keywords. We vary the number of extracted keywords per class $Z$ and show the results in Fig. 4. We can see that 1) since more keywords will hit more texts, more extracted keywords result in higher text coverage. 2) The change $(\Delta)$ of keywords falls below the threshold $\epsilon(0.1)$ in the $3^{\text {th }}$ update for all three keywords number settings. We can assume that $Z$ has little effect on the number of iterations for model convergence. 3) Increasing the number of keywords from 50 to 100 brings a great performance improvement, while more keywords $(Z=300)$ make little change.

\subsubsection{Effect of the IDF Power $M$}

We check the effect of the power of IDF $M$ in Eq. (9) by changing its value from 1 to 7 for ex- 


\begin{tabular}{c|cccccc}
\hline Metrics & Classifier / Iter & 1 & 2 & 3 & 4 & 5 \\
\hline \multirow{2}{*}{ Micro-F1 } & HAN & 0.69 & 0.71 & 0.72 & 0.72 & 0.72 \\
& Longformer & 0.69 & 0.77 & 0.78 & 0.78 & 0.78 \\
\hline \multirow{2}{*}{ Macro-F1 } & HAN & 0.68 & 0.70 & 0.70 & 0.71 & 0.71 \\
& Longformer & 0.68 & 0.76 & 0.77 & 0.76 & 0.77 \\
\hline
\end{tabular}

Table 5: Results of different classifier implementations.

tracting keywords, based on which we train the subgraph annotator and report the micro-f1 of subgraph annotation and the coverage of unlabeled texts. Results of the $1^{\text {th }}$ and $2^{\text {th }}$ iterations are shown in Fig. 5. With the increase of $M$, the accuracy of labeling also increases, but the coverage decreases. This is due to that a larger $M$ makes the algorithm extract more uncommon words, thus improving the accuracy while reducing the coverage.
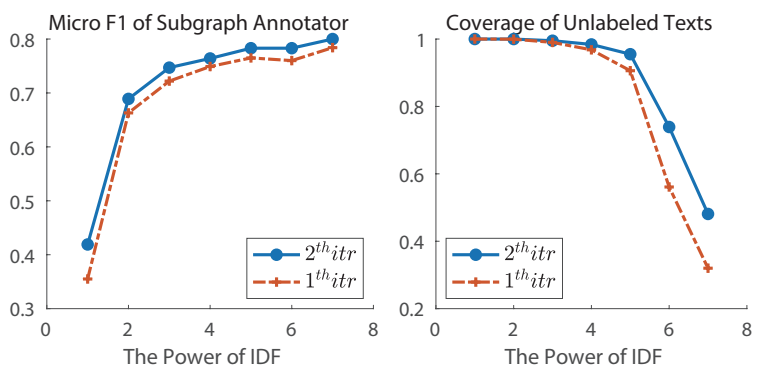

Figure 5: Effect of the IDF power $M$ on micro-f1 of subgraph annotation and coverage of unlabeled texts.

\subsubsection{Effect of Text Classifier Implementation}

Our framework is compatible with any classifier. Here, we replace the Longformer classifier (Beltagy et al., 2020) for long texts with HAN (Pappas and Popescu-Belis, 2017) classifier. Results are shown in Table 5. As we can see, our framework with HAN classifier still achieves good performance, surpassing SOTA by $7 \%$ in micro/macro-f1.

\subsubsection{Effects of Hyperparameters}

Here, we check the effects of two hyperparameters: the number of GIN layers and the number of epochs for finetuning subgraph annotator. The results of the $1^{\text {th }}$ and $2^{\text {th }}$ iterations are shown in Fig. 6. We can see that the accuracy of subgraph annotator decreases slightly as the number of GIN layers increases, which may be due to over smoothing. As finetuning goes, the labeling accuracy decreases slightly, which may be caused by overfitting.

\subsection{Case Study}

Here we present a case study to show the power of our framework. We take the technology class in AG
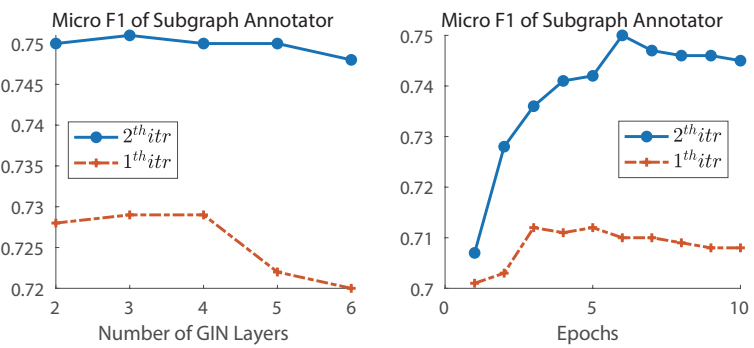

Figure 6: Hyperparameter effect.

News dataset as an example. In the beginning, we take "technology" as the initial keyword. At the end of the 1st/2nd iteration, the keywords are updated and the top 12 keywords are presented in Table 6 . Obviously, all the 12 keywords extracted by our method are correct, belonging to "technology" category. Furthermore, we check the annotation results in the 2nd iteration. Some annotations are shown in Table 7. We can see that our annotator gets more accurate pseudo-labels than keyword counting.

\begin{tabular}{cc}
\hline Iter & Top 12 Keywords \\
\hline 0 & technology \\
\hline 1 & $\begin{array}{c}\text { linux, yahoo, font, mars, nokia, napster } \\
\text { peer, adobe, titan, skype, cisco, google }\end{array}$ \\
\hline 2 & $\begin{array}{c}\text { linux, yahoo, font, mars, nokia, adobe } \\
\text { titan, skype, abbott, ibm, apple, xbox }\end{array}$ \\
\hline
\end{tabular}

Table 6: The top 12 keywords of technology class.

\begin{tabular}{c|ccc}
\hline Sentences & Count $\mathcal{A}$ GT \\
\hline $\begin{array}{c}\text { Google IPO Auction Off } \\
\text { to Rocky Start. WASHINGTON ... }\end{array}$ & 3 & 2 & 2 \\
$\begin{array}{c}\text { Strippers and pole dancers should be } \\
\text { banned from performing in stretch... } \\
\begin{array}{c}\text { The Olympics - weapon of mass distraction. } \\
\text { As Australians watch their country... }\end{array}\end{array}$ & 1 & 0 & 0 \\
\hline
\end{tabular}

Table 7: Some cases that our annotator $(\mathcal{A})$ outperforms keyword counting (Count). 'GT': Ground True. ' 0 ': politics, '1': sports, '2': business, '3': technology.

\section{Conclusion}

In this work, we propose a novel method for weakly-supervised text classification by exploiting the correlation among keywords. Our method follows an iterative paradigm. In each iteration, we first build a keyword graph and the task of assigning pseudo-labels is transformed into annotating keyword subgraphs. To accurately annotate subgraphs, we first train the subgraph annotator with a designed pretext task and then finetune it. The trained 
subgraph annotator is used to generate pseudo labels, with which we train a text classifier. Finally, we re-extract keywords from the classification results of the classifier. Our experiments on both long and short text datasets show that our method outperforms the existing ones. As for future work, we will focus on improving the proposed method by new mechanisms and network structures.

Acknowledgement. This work was supported by Alibaba Group through Alibaba Innovative Research Program.

\section{References}

Eugene Agichtein and Luis Gravano. 2000. Snowball: Extracting relations from large plain-text collections. In Proceedings of the fifth ACM conference on Digital libraries, pages 85-94.

Sonia Badene, Kate Thompson, Jean-Pierre Lorré, and Nicholas Asher. 2019. Data programming for learning discourse structure. In Proceedings of the 57th Annual Meeting of the Association for Computational Linguistics, pages 640-645, Florence, Italy. Association for Computational Linguistics.

Iz Beltagy, Matthew E. Peters, and Arman Cohan. 2020. Longformer: The long-document transformer. arXiv:2004.05150.

Ming-Wei Chang, Lev-Arie Ratinov, Dan Roth, and Vivek Srikumar. 2008. Importance of semantic representation: Dataless classification. In Proceedings of the AAAI Conference on Artificial Intelligence, volume 2, pages 830-835.

Jacob Devlin, Ming-Wei Chang, Kenton Lee, and Kristina Toutanova. 2019. BERT: Pre-training of deep bidirectional transformers for language understanding. In Proceedings of the 2019 Conference of the North American Chapter of the Association for Computational Linguistics: Human Language Technologies, Volume 1 (Long and Short Papers), pages 4171-4186, Minneapolis, Minnesota. Association for Computational Linguistics.

Evgeniy Gabrilovich, Shaul Markovitch, et al. 2007. Computing semantic relatedness using wikipediabased explicit semantic analysis. In International Joint Conference on Artificial Intelligence, volume 7, pages $1606-1611$.

Kaiming He, Haoqi Fan, Yuxin Wu, Saining Xie, and Ross Girshick. 2020. Momentum contrast for unsupervised visual representation learning. In Proceedings of the IEEE/CVF Conference on Computer Vision and Pattern Recognition, pages 9729-9738.

Weihua Hu, Bowen Liu, Joseph Gomes, Marinka Zitnik, Percy Liang, Vijay Pande, and Jure Leskovec. 2020a. Strategies for pre-training graph neural networks. In International Conference on Learning Representations.
Ziniu Hu, Yuxiao Dong, Kuansan Wang, Kai-Wei Chang, and Yizhou Sun. 2020b. Gpt-gnn: Generative pre-training of graph neural networks. In Proceedings of the 26th ACM SIGKDD International Conference on Knowledge Discovery \& Data Mining, pages 1857-1867.

Y. Jiao, Y. Xiong, J. Zhang, Y. Zhang, T. Zhang, and Y. Zhu. 2020. Sub-graph contrast for scalable self-supervised graph representation learning. In 2020 IEEE International Conference on Data Mining (ICDM), pages 222-231, Los Alamitos, CA, USA. IEEE Computer Society.

Thomas N. Kipf and Max Welling. 2017. Semisupervised classification with graph convolutional networks. In International Conference on Learning Representations (ICLR).

Benjamin J Kuipers, Patrick Beeson, Joseph Modayil, and Jefferson Provost. 2006. Bootstrap learning of foundational representations. Connection Science, 18(2):145-158.

Zhenzhong Lan, Mingda Chen, Sebastian Goodman, Kevin Gimpel, Piyush Sharma, and Radu Soricut. 2020. Albert: A lite bert for self-supervised learning of language representations. In International Conference on Learning Representations.

Jens Lehmann, Robert Isele, Max Jakob, Anja Jentzsch, Dimitris Kontokostas, Pablo Mendes, Sebastian Hellmann, Mohamed Morsey, Patrick Van Kleef, Sören Auer, and Christian Bizer. 2014. Dbpedia a large-scale, multilingual knowledge base extracted from wikipedia. Semantic Web Journal, 6.

Mike Lewis, Yinhan Liu, Naman Goyal, Marjan Ghazvininejad, Abdelrahman Mohamed, Omer Levy, Veselin Stoyanov, and Luke Zettlemoyer. 2020. BART: Denoising sequence-to-sequence pretraining for natural language generation, translation, and comprehension. In Proceedings of the 58th Annual Meeting of the Association for Computational Linguistics, pages 7871-7880, Online. Association for Computational Linguistics.

Ilya Loshchilov and Frank Hutter. 2019. Decoupled weight decay regularization. In International Conference on Learning Representations.

Andrew L. Maas, Raymond E. Daly, Peter T. Pham, Dan Huang, Andrew Y. Ng, and Christopher Potts. 2011. Learning word vectors for sentiment analysis. In Proceedings of the 49th Annual Meeting of the Association for Computational Linguistics: Human Language Technologies, pages 142-150, Portland, Oregon, USA. Association for Computational Linguistics.

Julian McAuley and Jure Leskovec. 2013. Hidden factors and hidden topics: Understanding rating dimensions with review text. In Proceedings of the 7th ACM Conference on Recommender Systems, RecSys '13, page 165-172, New York, NY, USA. Association for Computing Machinery. 
Dheeraj Mekala and Jingbo Shang. 2020. Contextualized weak supervision for text classification. In Proceedings of the 58th Annual Meeting of the Association for Computational Linguistics, pages 323-333, Online. Association for Computational Linguistics.

Yu Meng, Jiaming Shen, Chao Zhang, and Jiawei Han. 2018. Weakly-supervised neural text classification. In Proceedings of the 27th ACM International Conference on Information and Knowledge Management, pages 983-992.

Yu Meng, Jiaming Shen, Chao Zhang, and Jiawei Han. 2019. Weakly-supervised hierarchical text classification. In Proceedings of the AAAI Conference on Artificial Intelligence, volume 33, pages 6826-6833.

Yu Meng, Yunyi Zhang, Jiaxin Huang, Chenyan Xiong, Heng Ji, Chao Zhang, and Jiawei Han. 2020. Text classification using label names only: A language model self-training approach. In Proceedings of the 2020 Conference on Empirical Methods in Natural Language Processing (EMNLP), pages 9006-9017, Online. Association for Computational Linguistics.

Tomas Mikolov, Kai Chen, Greg Corrado, and Jeffrey Dean. 2013. Efficient estimation of word representations in vector space. In International Conference on Learning Representations (ICLR).

Mehdi Noroozi and Paolo Favaro. 2016. Unsupervised learning of visual representations by solving jigsaw puzzles. In European conference on computer vision, pages 69-84. Springer.

Nikolaos Pappas and Andrei Popescu-Belis. 2017. Multilingual hierarchical attention networks for document classification. In Proceedings of the Eighth International Joint Conference on Natural Language Processing (Volume 1: Long Papers), pages 1015-1025, Taipei, Taiwan. Asian Federation of Natural Language Processing.

Deepak Pathak, Philipp Krahenbuhl, Jeff Donahue, Trevor Darrell, and Alexei A Efros. 2016. Context encoders: Feature learning by inpainting. In Proceedings of the IEEE conference on computer vision and pattern recognition, pages 2536-2544.

Jiezhong Qiu, Qibin Chen, Yuxiao Dong, Jing Zhang, Hongxia Yang, Ming Ding, Kuansan Wang, and Jie Tang. 2020. Gcc: Graph contrastive coding for graph neural network pre-training. In Proceedings of the 26th ACM SIGKDD International Conference on Knowledge Discovery \& Data Mining, pages 1150-1160.

Alexander Ratner, Christopher De Sa, Sen Wu, Daniel Selsam, and Christopher Ré. 2016. Data programming: Creating large training sets, quickly. $A d$ vances in neural information processing systems, 29:3567.

Alexander J. Ratner, Stephen H. Bach, Henry R. Ehrenberg, and Christopher Ré. 2017. Snorkel: Fast training set generation for information extraction. In SIGMOD Conference, pages 1683-1686.
Ellen Riloff, Janyce Wiebe, and Theresa Wilson. 2003. Learning subjective nouns using extraction pattern bootstrapping. In Proceedings of the Seventh Conference on Natural Language Learning at HLTNAACL 2003, pages 25-32.

Yu Rong, Yatao Bian, Tingyang Xu, Weiyang Xie, Ying WEI, Wenbing Huang, and Junzhou Huang. 2020. Self-supervised graph transformer on largescale molecular data. In Advances in Neural Information Processing Systems, volume 33, pages 12559-12571. Curran Associates, Inc.

Chuck Rosenberg, Martial Hebert, and Henry Schneiderman. 2005. Semi-supervised self-training of object detection models. In 2005 Seventh IEEE Workshops on Applications of Computer Vision (WACV/MOTION'05) - Volume 1, volume 1, pages 29-36.

Jiaming Shen, Wenda Qiu, Yu Meng, Jingbo Shang, Xiang Ren, and Jiawei Han. 2021. TaxoClass: Hierarchical multi-label text classification using only class names. In Proceedings of the 2021 Conference of the North American Chapter of the Association for Computational Linguistics: Human Language Technologies, pages 4239-4249, Online. Association for Computational Linguistics.

Kai Shu, Subhabrata Mukherjee, Guoqing Zheng, Ahmed Hassan Awadallah, Milad Shokouhi, and Susan Dumais. 2020. Learning with weak supervision for email intent detection. In Proceedings of the 43rd International ACM SIGIR Conference on Research and Development in Information Retrieval, pages 1051-1060.

Yangqiu Song and Dan Roth. 2014. On dataless hierarchical text classification. In Proceedings of the AAAI Conference on Artificial Intelligence, volume 28.

Fangbo Tao, Chao Zhang, Xiusi Chen, Meng Jiang, Tim Hanratty, Lance Kaplan, and Jiawei Han. 2015. Doc2cube: Automated document allocation to text cube via dimension-aware joint embedding. Dimension, 2016:2017.

Petar Veličković, Guillem Cucurull, Arantxa Casanova, Adriana Romero, Pietro Liò, and Yoshua Bengio. 2018. Graph attention networks. In International Conference on Learning Representations.

Zihan Wang, Dheeraj Mekala, and Jingbo Shang. 2021. X-class: Text classification with extremely weak supervision. In Proceedings of the 2021 Conference of the North American Chapter of the Association for Computational Linguistics: Human Language Technologies, pages 3043-3053, Online. Association for Computational Linguistics.

Keyulu $\mathrm{Xu}$, Weihua $\mathrm{Hu}$, Jure Leskovec, and Stefanie Jegelka. 2019. How powerful are graph neural networks? In International Conference on Learning Representations. 
Wenpeng Yin, Jamaal Hay, and Dan Roth. 2019. Benchmarking zero-shot text classification: Datasets, evaluation and entailment approach. In Proceedings of the 2019 Conference on Empirical Methods in Natural Language Processing and the 9th International Joint Conference on Natural Language Processing (EMNLP-IJCNLP), pages 3914-3923, Hong Kong, China. Association for Computational Linguistics.

Xiang Zhang, Junbo Zhao, and Yann LeCun. 2015. Character-level convolutional networks for text classification. In Advances in Neural Information Processing Systems, volume 28. Curran Associates, Inc. 